\title{
High-temperature relaxor ferroelectric behavior in $\mathrm{Pr}$-doped $\mathrm{SrTiO}_{3}$
}

\author{
Rajeev Ranjan, ${ }^{1,2}$ Rudi Hackl, ${ }^{3}$ Amreesh Chandra, ${ }^{4}$ Elmar Schmidbauer, ${ }^{5}$ Dmytro Trots, ${ }^{6}$ and Hans Boysen ${ }^{2}$ \\ ${ }^{1}$ School of Materials Science and Technology, Institute of Technology, Banaras Hindu University, Varanasi-221005, India \\ ${ }^{2}$ Department fur Geo-und Umweltwissenschaften, Sektion Kristallographie, Ludwig Maximilians Universität, Am Coulombwall 1, 85748 \\ Garching, München, Germany \\ ${ }^{3}$ Walther Meissner Institute, Bayerische Akademie der Wissenschaften, 85748 Garching, München, Germany \\ ${ }^{4}$ Max Planck Institute for Polymer Research, Ackermannweg 10, 55128, Mainz, Germany \\ ${ }^{5}$ Department of Earth and Environmental Sciences, Sektion Geophysik, Ludwig Maximilians Universität München, Theresienstr. 41, \\ 80333 München, Germany \\ ${ }^{6}$ Hasylab/DESY, Notkestr. 85, 22607 Hamburg, Germany
}

(Received 20 July 2007; revised manuscript received 2 November 2007; published 20 December 2007)

\begin{abstract}
Temperature-dependent Raman scattering, dielectric, and powder x-ray diffraction studies have been carried out on Pr-doped $\mathrm{SrTiO}_{3}$ ceramic samples. Activation of $\mathrm{TO}_{2}$ and $\mathrm{TO}_{4}$ polar modes indicated increasing degree of polar distortion by Pr doping. Dielectric measurements revealed that the system exhibits dielectric relaxation peaks at $\sim 500 \mathrm{~K}$. The softening tendency of the polar $\mathrm{TO}_{1}$ soft mode decreases with increasing $\operatorname{Pr}$ doping. $\mathrm{X}$-ray diffraction results show no evidence of symmetry breaking across the dielectric peak temperatures. The system exhibits features of high-temperature relaxor ferroelectrics.
\end{abstract}

DOI: 10.1103/PhysRevB.76.224109 PACS number(s): 77.22. $-\mathrm{d}$, 77.80.-e, 68.35.Rh, 63.20.-e

\section{INTRODUCTION}

$\mathrm{SrTiO}_{3}(\mathrm{STO})$ has been a model system for understanding the mechanism of structural phase transitions in solids. ${ }^{1}$ It is also a well known for its incipient ferroelectric and quantum paraelectric behavior at low temperatures. ${ }^{2}$ The relative dielectric permittivity $\left(\varepsilon_{r}^{\prime}\right)$ of bulk STO increases with decreasing temperature but saturates below $4 \mathrm{~K}$ presumably due to suppression of the ferroelectric state by zero-point quantum fluctuations of the lattice. ${ }^{2}$ The ferroelectric state can, however, be stabilized by application of an external electric field, ${ }^{3}$ uniaxial stress, ${ }^{4}$ and also by chemical and isotopic substitutions. ${ }^{5-7}$ An unexpected onset of an intermediate antiferroelectric phase has also been reported for $\mathrm{Ca}$ substitution in STO. ${ }^{8-10}$ In most of the cases the ferroelectric ordering occurs well below room temperature. For example, the temperature corresponding to the dielectric permittivity anomalies $\left(T_{\mathrm{m}}\right)$ in Ca-substituted samples has been reported to be less than $35 \mathrm{~K}^{5}$ In Bi-substituted STO, although dielectric relaxation peaks are reported at temperatures up to $\sim 200 \mathrm{~K}$, macroscopic polarization could survive only for temperatures less than $100 \mathrm{~K} .{ }^{11}$ High-temperature ferroelectric materials are of technological interest, and with increasing environmental concern, efforts are being made to search for more environmentally friendly lead-free ferroelectric materials. A possibility of stabilizing the ferroelectric phase at room temperature and above was theoretically predicted for biaxially strained $\mathrm{STO}^{12}$ and was subsequently realized in a thin STO film grown on a $\mathrm{DyScO}_{3}$ substrate. ${ }^{13}$ Durán et al. ${ }^{14}$ have recently reported the existence of a ferroelectric phase in $\mathrm{Pr}$-doped $\mathrm{SrTiO}_{3}$ ceramics even at room temperature. So far no detailed study in this regard has been reported. In order to have a better understanding of the development of the ferroelectric state in Pr-doped STO, we have carried out temperature-dependent Raman, dielectric, and x-ray diffraction measurements on this system. It is shown that the system exhibits dielectric relaxation around $500 \mathrm{~K}$ and the ferroelectric state reported at room temperature therefore arises due to freezing of dynamic polar clusters around giant electric dipoles formed by Pr substitution.

\section{EXPERIMENT}

Ceramic specimens of Pr-doped $\mathrm{SrTiO}_{3}$ were prepared as per the chemical formula $\mathrm{Sr}_{1-x} \mathrm{Pr}_{x} \mathrm{TiO}_{3}$ with $x=0.025,0.05$, and 0.075 by the standard solid-state reaction method. Stoichiometric ratios of $\mathrm{SrCO}_{3}, \mathrm{TiO}_{2}$, and $\mathrm{Pr}_{6} \mathrm{O}_{11}$, each of purity greater than $99.9 \%$ (Alpha Aesar), were thoroughly mixed and calcined at $1100{ }^{\circ} \mathrm{C}$ for $3 \mathrm{~h}$. The disk-shaped pellets were sintered at $1300{ }^{\circ} \mathrm{C}$ for $2 \mathrm{~h}$. It may, however, be mentioned that Pr can exist in $3+$ and $4+$ valence states, ${ }^{14}$ and since the sizes of $\operatorname{Pr}^{3+}(1.126 \AA)$ and $\operatorname{Pr}^{4+}(0.96 \AA)$ lie between that of $\mathrm{Sr}^{2+}(1.44 \AA)$ and $\mathrm{Ti}^{4+}(0.61 \AA),{ }^{15}$ a possibility of some fraction of the substituted Pr ions occupying the Ti site is also quite likely. In this paper, we have, however, not concerned ourselves with the problem of site occupancy of the substituted Pr ions in STO. Raman spectra were recorded on polished pellets in backscattering geometry. The samples were mounted on a heating stage with the temperature controllable in the range $300-800 \mathrm{~K}$. The power of the $\mathrm{Kr}^{+}$laser operated at $531 \mathrm{~nm}$ was set at $2 \mathrm{~mW}$. The spectra were recorded with a Horiba Jobin Yvon T64000 triple spectrometer equipped with a liquid- $\mathrm{N}_{2}$-cooled back-thinned chargecoupled-device (CCD) detector. High-resolution powder x-ray diffraction data were collected at HASYLAB (B2 beamline) at the synchrotron source (DESY) in Germany, using a wavelength of $0.79809 \AA$ (calibrated with a $\mathrm{LaB}_{6}$ pattern). For dielectric measurements platinum electrodes were applied on cleaned surfaces of the disk-shaped sintered pellets. Capacitance and loss tangent were measured using a HP 4192 impedance analyzer. 


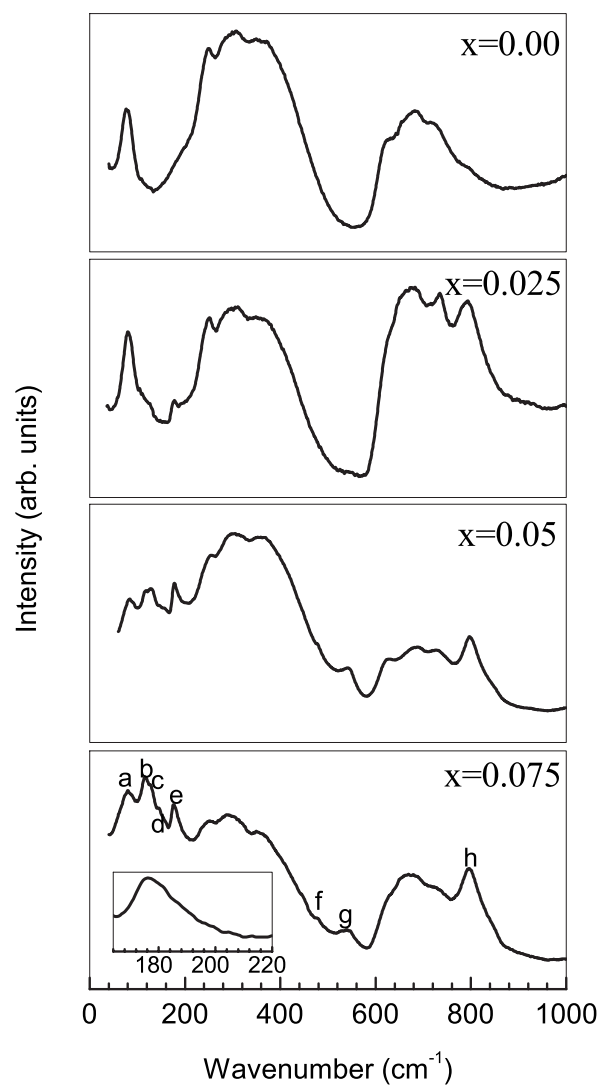

FIG. 1. Raman spectra of $\mathrm{Sr}_{1-x} \mathrm{Pr}_{x} \mathrm{TiO}_{3}$ for $x=0.00,0.025,0.05$, and 0.075 at $300 \mathrm{~K}$. The inset in the lowest panel shows magnified plot in a limited frequency range. For convenience, the various modes are labeled in alphabetical order in the lowest panel (see text).

\section{RESULTS AND DISCUSSION}

\section{A. Raman scattering at $300 \mathrm{~K}$ : Composition dependence}

Figure 1 shows the Raman spectra of $\mathrm{Sr}_{1-x} \mathrm{Pr}_{x} \mathrm{TiO}_{3}$ (SPT) for $x=0.00,0.025,0.05$, and 0.075 . Apart from the two second-order broadbands centered around 300 and $700 \mathrm{~cm}^{-1}$, distinct first-order modes are seen for all the compositions. These modes are labeled in alphabetical order $a-h$ for $x$ $=0.075$ in Fig. 1 and appear at $80(a), 117(b), 127(c), 145$ $(d), 175(e), 475(f), 540(g)$, and $795(h) \mathrm{cm}^{-1}$. All these Raman modes are, in principle, forbidden by selection rules in the cubic phase of STO. Their occurrence is made possible by relaxation of these rules by unintentional and intentional structural defects. By comparison with previously published Raman and infrared scattering results on bulk $\mathrm{STO}^{16-21}$ and on thin STO films ${ }^{22}$ we identified the four Raman modes near $175,475,540$, and $795 \mathrm{~cm}^{-1}$ as $\mathrm{TO}_{2}, \mathrm{LO}_{3}, \mathrm{TO}_{4}$, and $\mathrm{LO}_{4}$, modes, respectively. The appearance of $\mathrm{TO}_{2}$ and $\mathrm{TO}_{4}$ modes, has been considered as evidence of polar nanoregions (PNRs) which characterize a relaxor ferroelectric system. ${ }^{16,23,24}$ The intensities of the $\mathrm{TO}_{2}$ and $\mathrm{TO}_{4}$ modes are more pronounced for the compositions $x=0.05$ and 0.075 than for $x=0.025$, suggesting increasing degree of polar distortion for the two higher compositions. The line shape of the $\mathrm{TO}_{2}$ mode is strongly asymmetric and tails off on the high- frequency side (see inset in the lowest panel of Fig. 1). This effect, known as the Fano effect, has also been reported in Ca-doped STO, ${ }^{16}$ thin STO film, ${ }^{22}$ and in doped $\mathrm{KTaO}_{3}$ crystals. 23,24

A weak Raman mode at $\sim 145 \mathrm{~cm}^{-1}$, visible in the spectra of the $x=0.05$ and 0.075 samples, corresponds to the hard structural $E_{\mathrm{g}}$ mode. This mode becomes visible in doped and undoped STO below the cubic-tetragonal antiferrodistortive (AFD) structural phase transition temperature. ${ }^{16}$ The AFD transition in STO is driven by softening and freezing of a triply degenerate $R_{25}$ phonon at the $R$ point of the cubic Brillouin zone. ${ }^{25}$ The presence of this weak mode indicates local/global AFD distortion at room temperature. Local AFD distortions have also been reported at room temperature in the (globally) cubic phase of $\mathrm{Sr}_{0.94} \mathrm{Ca}_{0.06} \mathrm{TiO}_{3},{ }^{26}$ as well as in Bi-doped STO. ${ }^{27}$

A mode near $80 \mathrm{~cm}^{-1}$ is most pronounced for the compositions $x=0.00$ and 0.025 . This mode corresponds to an IRactive soft $\mathrm{TO}_{1}$ mode ${ }^{3,17,19}$ which is known to exhibit a softening tendency. ${ }^{3,16}$ Porokhonskyy et al. ${ }^{27}$ have shown that doping of impurity ions considerably influences the frequency of this mode. Bi doping increases the mode frequency (at $300 \mathrm{~K}$ ) to $103 \mathrm{~cm}^{-1}$ for $0.67 \mathrm{~mol} \% \mathrm{Bi}$ and to $126 \mathrm{~cm}^{-1}$ for $13.3 \mathrm{~mol} \%$ of Bi. This hardening has been attributed to frequency shift of anharmonic oscillator biased by random electric fields of the dipole impurities. In contrast to Bi-doped STO, the frequency of this mode at $300 \mathrm{~K}$ is nearly composition independent in Pr-doped STO. Further, two new modes near 117 and $127 \mathrm{~cm}^{-1}$ are observed. Unlike the other modes mentioned above, these new modes are not likely to originate from disorder-induced activation of the modes of cubic STO. Their presence is suggestive of activation of local modes by Pr doping. Similar modes at $\sim 261$ and $520 \mathrm{~cm}^{-1}$ in Bi-doped STO have been attributed to originate from coupling of the dynamics off-centered $\mathrm{Bi}$ ions with the soft TO mode. ${ }^{27}$

\section{B. Raman scattering: Temperature dependence}

Raman spectra as a function of temperature for all the three compositions were measured to understand the role of lattice dynamics in bringing about the ferroelectric state in Pr-doped STO. Figures 2 and 3 show Raman spectra of the samples with $x=0.025$ and 0.075 at selected temperatures. Of the various phonon modes mentioned above, only the lowestenergy phonon $\left(\mathrm{TO}_{1}\right)$ was found to be sensitive to changes in temperature. The hard polar modes $\mathrm{TO}_{2}$ and $\mathrm{TO}_{4}$ in doped incipient ferroelectrics such as STO and $\mathrm{KTaO}_{3}$ have been used to characterize the PNRs. ${ }^{23,24,28}$ The intensity of the $\mathrm{TO}_{2}$ mode in $\mathrm{Sr}_{0.993} \mathrm{Ca}_{0.007} \mathrm{TiO}_{3}\left(T_{\mathrm{m}} \sim 18 \mathrm{~K}\right)$ has been related to the space and time autocorrelation $\left\langle P^{2}\right\rangle$ of the fluctuating impurity-induced polarization $P(r, t) .{ }^{28}$ The intensity of the $\mathrm{TO}_{2}$ mode in Pr-doped STO persisted on heating up to $773 \mathrm{~K}$, which is $\sim 280 \mathrm{~K}$ above the temperature of the dielectric anomaly $\left(T_{\mathrm{m}} \sim 500 \mathrm{~K}\right)$, without any appreciable change. This suggests that the polar regions in Pr-doped STO persist up to $773 \mathrm{~K}$ and presumably even higher.

Figures 4(a) and 4(b) show magnified plots of the $\mathrm{TO}_{1}$ mode of $\mathrm{Sr}_{0.95} \mathrm{Pr}_{0.05} \mathrm{TiO}_{3}$ and $\mathrm{Sr}_{0.925} \mathrm{Ca}_{0.075} \mathrm{TiO}_{3}$, respectively, 


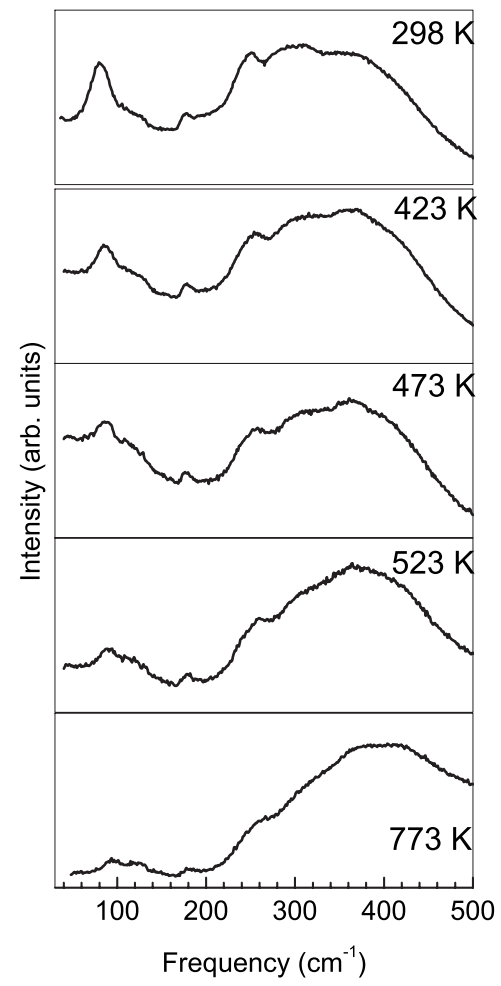

FIG. 2. Raman spectra of $\mathrm{Sr}_{0.975} \mathrm{Pr}_{0.025} \mathrm{TiO}_{3}$ at various temperatures

at three representative temperatures. The change in frequency of this mode with temperature is evident for $x$ $=0.05$. For $x=0.075$, the frequency of this mode is independent of temperature [Fig. 4(b)]. Figure 5 shows a typical variation of the frequency of the $\mathrm{TO}_{1}$ mode of $x=0.025$ with temperature. The frequency of this mode changes by $15 \mathrm{~cm}^{-1}$

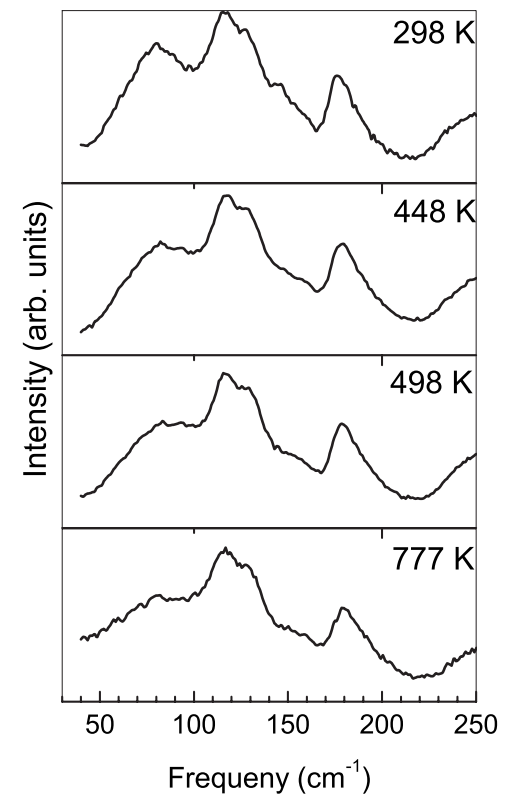

FIG. 3. Raman spectra of $\mathrm{Sr}_{0.925} \mathrm{Pr}_{0.075} \mathrm{TiO}_{3}$ at various temperatures
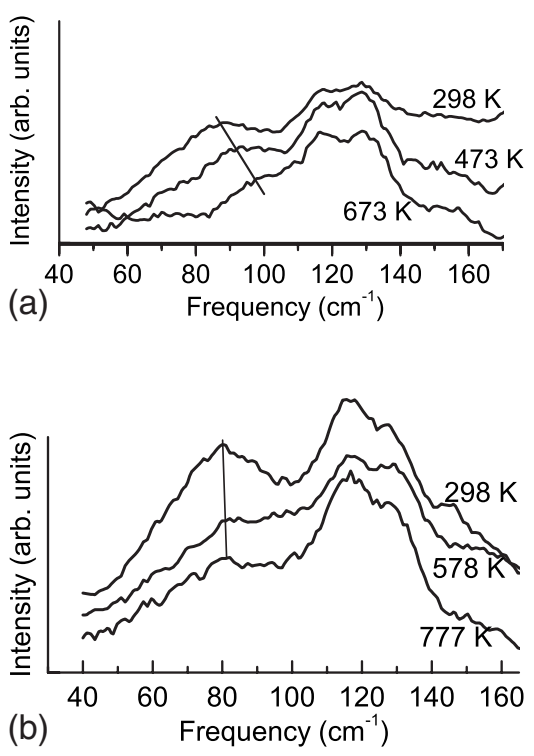

FIG. 4. Magnified plot of $\mathrm{TO}_{1}$ mode of (a) $\mathrm{Sr}_{0.95} \mathrm{Pr}_{0.05} \mathrm{TiO}_{3}$ and (b) $\mathrm{Sr}_{0.925} \mathrm{Pr}_{0.075} \mathrm{TiO}_{3}$ at three representative temperatures. The $\mathrm{TO}_{1}$ mode retains its softening tendency in (a) while it is almost temperature independent in (b).

on varying the temperature from $298 \mathrm{~K}$ to $773 \mathrm{~K}$. The softening ability of the $\mathrm{TO}_{1}$ mode gradually decreases with increasing the Pr content as is evident from Fig. 6 which depicts the total change in frequency on heating from $300 \mathrm{~K}$ to $773 \mathrm{~K}$ for the three compositions. For $x=0.075$, the $\mathrm{TO}_{1}$ mode behaves like a hard mode. Similar behavior was reported in Ref. 27 for Bi-doped STO. A comparison of results with Bi-doped STO suggests that the stiffening tendency of the soft $\mathrm{TO}_{1}$ mode is more enhanced in Pr-doped STO specimens. For example, for $0.67 \mathrm{~mol} \%$ Bi-doped STO, the mode frequency has been reported to change from $103 \mathrm{~cm}^{-1}$ at $300 \mathrm{~K}$ to $86 \mathrm{~cm}^{-1}$ at $260 \mathrm{~K}$-i.e., a shift of $17 \mathrm{~cm}^{-1}$ on changing the temperature by merely $40 \mathrm{~K}{ }^{27}$ The softening behavior of the $\mathrm{TO}_{1}$ mode in Bi-doped STO, though reduced on increasing the $\mathrm{Bi}$ concentration, survives even for $13.3 \mathrm{~mol} \%$ of $\mathrm{Bi}$ doping and continues well below $T_{\mathrm{m}} \cdot{ }^{27}$ Thus, unlike for the lightly Ca-doped STO $\left(\mathrm{Sr}_{0.993} \mathrm{Ca}_{0.007} \mathrm{TiO}_{3}, T_{\mathrm{m}} \sim 18 \mathrm{~K}\right),{ }^{28}$ where it has been shown that the $\mathrm{TO}_{1}$ mode hardens below $T_{\mathrm{m}}$ and splits into three components, thereby lowering the symmetry of the system from tetragonal to orthorhombic, ${ }^{16}$ the soft mode is not hard-

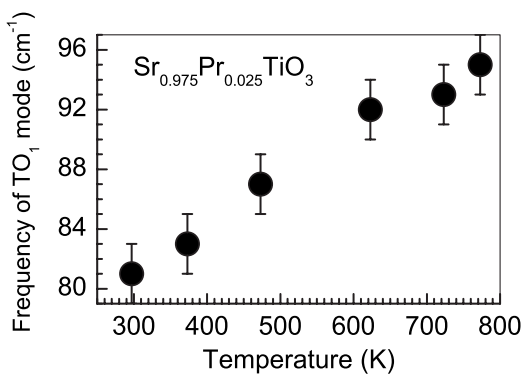

FIG. 5. Temperature variation of frequency of the $\mathrm{TO}_{1}$ mode of $\mathrm{Sr}_{0.975} \operatorname{Pr}_{0.025} \mathrm{TiO}_{3}$. 


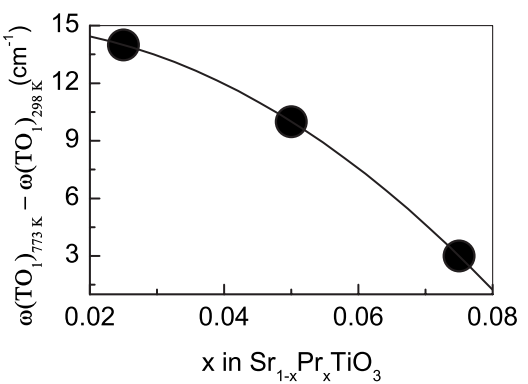

FIG. 6. Variation of the difference of the frequency of the $\mathrm{TO}_{1}$ mode at $298 \mathrm{~K}$ and $773 \mathrm{~K}$ with composition. The graph shows that Pr doping gradually decrease the softening ability of the $\mathrm{TO}_{1}$ mode.

ened below their respective $T_{\mathrm{m}}$ 's of Pr- and Bi-doped STO $(x=0.025,0.05)$. As a result, a symmetry change across $T_{\mathrm{m}}$ is less likely in the latter systems. All this evidence suggests that the dielectric anomalies reported in the Pr-doped STO samples ${ }^{14}$ cannot be due to a displacive ferroelectric phase transition.

\section{Dielectric response}

Having shown in the previous section that the reported dielectric anomalies in Pr-doped STO are not related to displacive ferroelectric transition, we investigated the dielectric behavior of the Pr-doped STO ceramic pellets as a function of temperature at various frequencies of the measuring ac signal. Figure 7 shows the real $\left(\varepsilon_{\mathrm{r}}^{\prime}\right)$ and imaginary $\left(\varepsilon_{\mathrm{r}}^{\prime \prime}\right)$ parts of the relative dielectric permittivity as a function of temperature at different frequencies. Two features worth noting are that (i) for a given frequency, the imaginary part peaks at

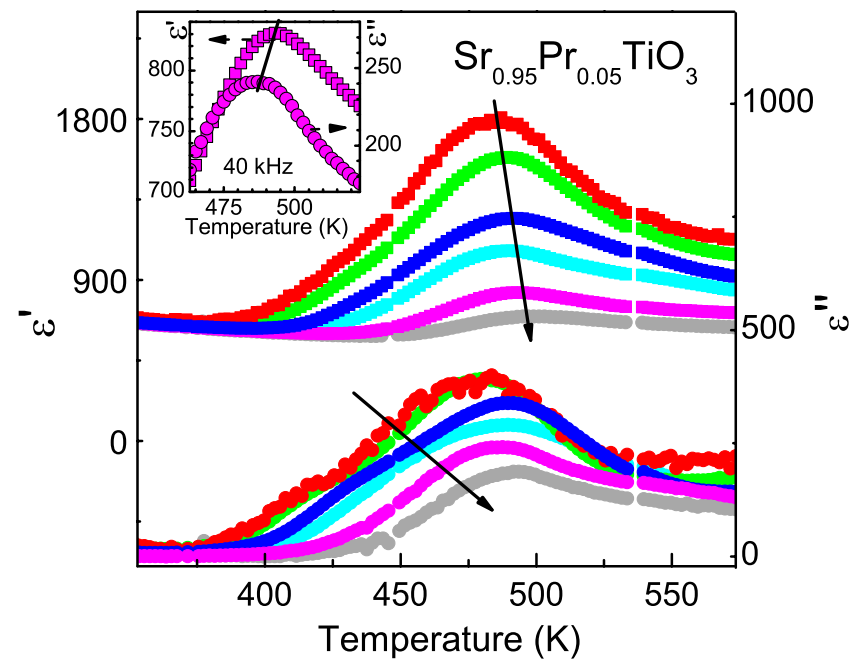

FIG. 7. (Color online) Temperature variation of real (upper plots) and imaginary parts (lower plots) of the relative dielectric permittivity at various frequencies: (a) $0.4 \mathrm{kHz}$, (b) $1 \mathrm{kHz}$, (c) $4 \mathrm{kHz}$ (d) $10 \mathrm{kHz}$, (e) $40 \mathrm{kHz}$, and (f) $100 \mathrm{kHz}$. The arrows indicate the direction of increasing frequency. The inset shows magnified plot of the real and imaginary parts of relative permittivity at $40 \mathrm{kHz}$. lower temperature than the real part (see inset of Fig. 7) and (ii) the peak temperature of both $\varepsilon_{\mathrm{r}}^{\prime \prime}$ and $\varepsilon_{\mathrm{r}}^{\prime \prime}$ shifts to higher temperature with increasing frequency of the measuring signal. The permittivity peaks therefore correspond to dielectric relaxation and not a phase transition. This is consistent with the Raman results reported in the previous section. Further, the fact that this composition exhibits polarization-electricfield $(P-E)$ hysteresis loop characteristic of a ferroelectric state, at room temperature, ${ }^{14}$ suggests that the observed dielectric relaxation is a manifestation of relaxor ferroelectric behavior. $^{29,30}$ Relaxor ferroelectric behavior below room temperature has also been reported in Bi-doped STO. ${ }^{11}$ An attempt to fit Vogel-Fulcher relationship to the peak temperatures (obtained by visual inspection) at different frequencies was however unsuccessful. A close inspection of the shape of the peaks revealed that more than one relaxation processes is at play. In this context it is interesting to note that the dielectric relaxation above room temperature in the imaginary part of permittivity, attributed to Maxwell-Wagner relaxation, has been reported even in pure STO ceramics. ${ }^{31}$ The asymmetric relaxation peaks in the imaginary part of the permittivity observed in Pr-doped STO can therefore have contributions from the Maxwell-Wagner process as well. Further, in the present case, since divalent $\mathrm{Sr}^{2+}$ is replaced by $\mathrm{Pr}$ which can exist in 3+ as well as 4+ states, strontium and oxygen vacancies will be created to satisfy charge neutrality. The existence of $\mathrm{Pr}^{3+}$ and $\mathrm{Pr}^{4+}$ valence states at the $\mathrm{Sr}$ sites and formation of $\mathrm{Pr}^{3+} / \mathrm{Pr}^{4+}-V_{\mathrm{Sr}}$ centers responsible for setting up random (local) electric field and/or strain fields has been proposed in Ref. 14. Such a random field can induce a ferroelectric domain state and give rise to a relaxor ferroelectric behavior. ${ }^{32}$

As far as our present understanding is concerned, the development of a ferroelectric state in doped incipient ferroelectric systems is intimately related to the formation of PNRs around the defect sites, the size of which grow on approaching $T_{\mathrm{m}}$ from the high-temperature side due to coupling with the soft ferroelectric mode. In this scenario, the size of a PNR should be determined by the magnitude of the dipole moment of the dipolar species in the host matrix. At any given temperature, a dipole with larger moment will polarize the surrounding host matrix more than a dipole with smaller moment. Since the dielectric permittivity of STO is considerably lower above room temperature as compared to its value at low temperatures, ${ }^{2}$ freezing of polar clusters above room temperature and consequent development of a ferroelectric state suggests the presence of dipolar species with giant dipole moments at the defect sites. These giant dipoles are able to create polar clusters (PNRs) of sufficiently large size even at high temperatures such that they can overlap and eventually lead to the development of a ferroelectric state. In analogy with the previous studies on Ca-doped STO, ${ }^{32}$ the smaller size of $\operatorname{Pr}^{3+}(1.126 \AA)$ and $\operatorname{Pr}^{4+}$ $(0.96 \AA)$ than that of $\mathrm{Sr}^{2+}(1.44 \AA)$ can cause $\mathrm{Pr}^{3+} / \mathrm{Pr}^{4+}$ to take up off-centered positions and create electric dipoles around which PNRs can develop. Alternatively, it is also possible for $\mathrm{Ti}^{4+}$ near a Pr site to take up an off-centered position, resulting in local ferroelectric distortions, due to favorable hybridization between Ti $d$ orbitals and $\mathrm{O} 2 p$ orbitals $^{33}$ assisted by local strain induced by size mismatch between the host and guest ions. Skanavi et al. have considered hop- 


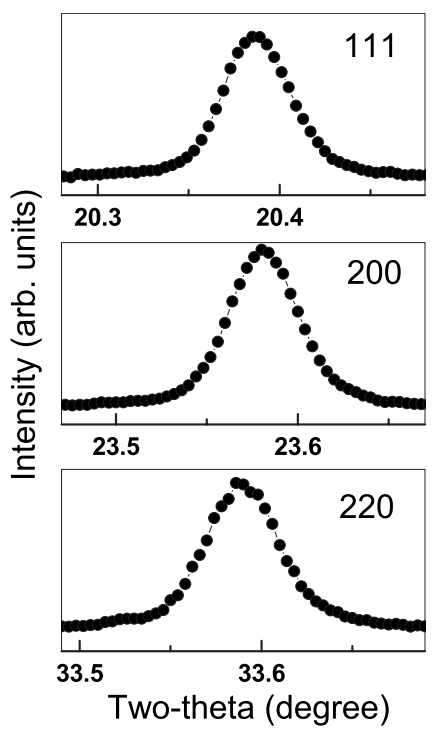

FIG. 8. Powder x-ray diffraction profiles of (a) $\{111\}$, (b) $\{200\}$, and (c) $\{220\}$ cubic Bragg reflections of $\mathrm{Sr}_{0.95} \mathrm{Pr}_{0.05} \mathrm{TiO}_{3}$.

ping of off-centered $\mathrm{Ti}^{4+}$ to explain the dielectric relaxation in Bi-doped STO. ${ }^{34}$ Local structure study can reveal the correct picture among these plausible models.

\section{X-ray powder diffraction}

In order to investigate whether or not the development of the ferroelectric state is accompanied by breaking of the global symmetry we carried out high resolution powder x-ray diffraction at room temperature for all the compositions mentioned above. Since STO is cubic $(P m \overline{3} m)$ at $300 \mathrm{~K}$, all the Bragg peaks must be singlet. Any distortion of the cubic lattice would result in the development of an asymmetry or splitting of the some of the Bragg reflections. Figure 8 shows selected profiles corresponding to the Bragg reflections $\{111\},\{200\}$, and $\{220\}$ of $\mathrm{Sr}_{0.95} \mathrm{Pr}_{0.05} \mathrm{TiO}_{3}$ at room temperature. It is evident from this figure that all the profiles are singlets, thereby suggesting that there is no noticeable distortion of the cubic lattice in the ferroelectric state. The present case is more akin to the well-known high-temperature relaxor $\mathrm{PbMg}_{1 / 3} \mathrm{Nb}_{2 / 3} \mathrm{O}_{3}\left(\mathrm{PMN}\right.$ ), and $\mathrm{Li}$-doped $\mathrm{KTaO}_{3}$ (for $\mathrm{Li}$ concentration less than $5 \mathrm{~mol} \%$ ) systems. Both systems do not exhibit features of global symmetry breaking below their respective $T_{\mathrm{m}}$ 's. ${ }^{35-37}$

\section{CONCLUSIONS}

To summarize, we have shown that $\mathrm{Pr}$ doping in $\mathrm{SrTiO}_{3}$ $\left(\mathrm{Sr}_{1-x} \mathrm{Pr}_{x} \mathrm{TiO}_{3}\right)$ exhibits dielectric anomalies around $500 \mathrm{~K}$, a temperature which is considerably large than those reported for other impurity systems in STO. The polar $\mathrm{TO}_{1}$ mode behaves differently for different compositions. For $x=0.025$ and 0.05 it behaves like a soft mode while it is almost temperature independent for $x=0.075$. The softening behavior in the first two compositions continues well below $T_{\mathrm{m}}$, suggesting that the dielectric anomalies in this system and the related development of ferroelectric state below $T_{\mathrm{m}}$ are not due to a displacive ferroelectric phase transition proposed earlier. The global structure of the system well below the permittivity anomaly temperature $\left(T_{\mathrm{m}}\right)$ remains cubic, suggesting that the global lattice distortion in the ferroelectric state is insignificant. Detailed dielectric measurements have revealed that the dielectric anomalies are related to relaxational freezing of PNRs similar to other relaxor ferroelectric systems. It is proposed that Pr doping induces "giant electric dipoles" which are able to polarize the $\mathrm{SrTiO}_{3}$ matrix around them and lead to their cooperative freezing without the need to lower the temperature of the system well below $300 \mathrm{~K}$. The results are expected to stimulate further research in this direction and may lead to the discovery of other systems based on doped STO which exhibit ferroelectric behavior at high temperatures that may have important technological implications.

\section{ACKNOWLEDGMENTS}

This work was supported by the Department of Science and Technology, Government of India. R.R. is grateful to the Alexander von Humboldt foundation for support during a stay in Germany during which part of this work was carried out.
${ }^{1}$ A. D. Bruce and R. A. Cowley, Adv. Phys. 29, 219 (1980).

${ }^{2}$ K. A. Müller and H. Burkard, Phys. Rev. B 19, 3593 (1979).

${ }^{3}$ P. A. Fleury and J. M. Worlock, Phys. Rev. 174, 613 (1968).

${ }^{4}$ H. Uwe and T. Sakudo, Phys. Rev. B 13, 271 (1976).

${ }^{5}$ J. G. Bednorz and K. A. Müller, Phys. Rev. Lett. 52, 2289 (1984).

${ }^{6}$ V. V. Lemanov, E. P. Smirnova, P. P. Syrnikov, and E. A. Tarakanov, Phys. Rev. B 54, 3151 (1996).

${ }^{7}$ M. Itoh, R. Wang, Y. Inaguma, T. Yamaguchi, Y.-J. Shan, and T. Nakamura, Phys. Rev. Lett. 82, 3540 (1999).

${ }^{8}$ R. Ranjan, D. Pandey, and N. P. Lalla, Phys. Rev. Lett. 84, 3726 (2000)

${ }^{9}$ R. Ranjan and D. Pandey, J. Phys.: Condens. Matter 13, 4239 (2001)
${ }^{10}$ R. Ranjan and D. Pandey, J. Phys.: Condens. Matter 13, 4251 (2001).

${ }^{11}$ Chen Ang, Zhi Yu, P. Lunkenheimer, J. Hemberger, and A. Loidl, Phys. Rev. B 59, 6670 (1999).

${ }^{12}$ N. A. Pertsev, A. K. Tagantsev, and N. Setter, Phys. Rev. B 61, R825 (2000).

${ }^{13}$ J. H. Haeni, P. Irvin, W. Chang, R. Uecker, P. Reiche, Y. L. Li, S. Choudhury, W. Tian, M. E. Hawley, B. Craigo, A. K. Tagantsev, X. Q. Pan, S. K. Streiffer, L. Q. Chen, S. W. Kirchoefer, J. Levy, and D. G. Schlom, Nature (London) 430, 758 (2004).

${ }^{14}$ A. Durán, E. Martínez, J. A. Díaz, and J. M. Siqueiros, J. Appl. Phys. 97, 104109 (2005).

${ }^{15}$ R. D. Shannon, Acta Crystallogr., Sect. A: Cryst. Phys., Diffr., 
Theor. Gen. Crystallogr. 32, 751 (1976).

${ }^{16}$ U. Bianchi, W. Kleemann, and J. G. Bednorz, J. Phys.: Condens. Matter 6, 1229 (1994).

${ }^{17}$ J. Petzelt, T. Ostapchuk, I. Gregora, I. Rychetský, S. HoffmannEifert, A. V. Pronin, Y. Yuzyuk, B. P. Gorshunov, S. Kamba, V. Bovtun, J. Pokorný, M. Savinov, V. Porokhonskyy, D. Rafaja, P. Vaněk, A. Almeida, M. R. Chaves, A. A. Volkov, M. Dressel, and R. Waser, Phys. Rev. B 64, 184111 (2001).

${ }^{18}$ V. N. Denisov, B. N. Mavrin, and V. B. Podobedov, Phys. Rep. 151, 1 (1987).

${ }^{19}$ H. Vogt and G. Rossbroich, Phys. Rev. B 24, 3086 (1981).

${ }^{20}$ W. G. Nielsen and J. G. Skinner, J. Chem. Phys. 48, 2240 (1968).

${ }^{21}$ A. S. Barker, Jr., Phys. Rev. 145, 391 (1966).

${ }^{22}$ A. A. Sirenko, I. A. Akimov, J. R. Fox, A. M. Clark, H. C. Li, W. Si, and X. X. Xi, Phys. Rev. Lett. 82, 4500 (1999).

${ }^{23}$ J. Toulouse, P. DiAntonio, B. E. Vugmeister, X. M. Wang, and L. A. Knauss, Phys. Rev. Lett. 68, 232 (1992).

${ }^{24}$ P. DiAntonio, B. E. Vugmeister, J. Toulouse, and L. A. Boatner, Phys. Rev. B 47, 5629 (1993).

${ }^{25}$ P. A. Fleury, J. F. Scott, and J. M. Worlock, Phys. Rev. Lett. 21, $16(1968)$
${ }^{26}$ S. K. Mishra, R. Ranjan, D. Pandey, P. Ranson, R. Ouillon, J. P. Lucarre, and P. Pruzan, J. Solid State Chem. 178, 2846 (2005).

${ }^{27}$ V. Porokhonskyy, A. Pashkin, V. Bovtun, J. Petzelt, M. Savinov, P. Samoukhina, T. Ostapchuk, J. Pokorny, M. Avdeev, A. Kholkin, and P. Vilarinho, Phys. Rev. B 69, 144104 (2004).

${ }^{28}$ U. Bianchi, J. Dec, W. Kleemann, and J. G. Bednorz, Phys. Rev. B 51, 8737 (1995).

${ }^{29}$ L. E. Cross, Ferroelectrics 76, 241 (1987).

${ }^{30}$ G. A. Samara, J. Phys.: Condens. Matter 15, R367 (2003).

${ }^{31}$ H. Neumann and G. Arlt, Ferroelectrics 69, 179 (1986).

${ }^{32}$ W. Kleemann, Int. J. Mod. Phys. B 7, 2469 (1993).

${ }^{33}$ R. E. Cohen, Nature (London) 358, 136 (1992).

${ }^{34}$ G. I. Scanavi, I. J. Ksendzov, V. A. Trigubenko, and V. G. Prokhvatilov, Zh. Eksp. Teor. Fiz. 33, 320 (1957) [Sov. Phys. JETP 6, 250 (1958)].

${ }^{35} \mathrm{~N}$. de Mathan, E. Husson, G. Calvarin, J. R. Gavarri, A. W. Hewat, and A. Morell, J. Phys.: Condens. Matter 3, 8158 (1991).

${ }^{36}$ S. R. Andrews, J. Phys. C 18, 1357 (1985).

${ }^{37}$ U. T. Hochli, K. Knorr, and A. Loidl, Adv. Phys. 39, 405 (1990). 